\title{
Numerical Analysis of Mixed-Phase Icing Cloud Simulations in the NASA Propulsion Systems Laboratory
}

Tadas Bartkus, Jen-Ching Tsao

Ohio Aerospace Institute

Peter Struk, Judith Van Zante

NASA Glenn Research Center

Aviation, 13-17 June 2016

Washington, D.C. 


\section{Outline}

- Introduction

- Model Formulation

- Sample Simulation

- Model/Experiment Comparisons

- Preliminary Tests to Fundamental ICI - Run May 2015 PSL

- 3 Sweeps (TWC, RH, $\mathrm{T}_{\text {water }}$ )

- Particle size comparison

- Summary 


\section{Introduction}

- Many engine power-loss events reported since the 1990's

- Mason et al. hypothesized how power-loss events can result from ice crystals entering the engine core

- Ingestion of ice into engine is studied at NASA PSL and elsewhere

- Observed environmental conditions changed with cloud activation

- Gas temperature change

- Humidity change

- Hypothesis: Thermal interaction between air and cloud

- Model previously written to simulate NRC RATFac

- Objective: Understand the air - cloud interactions in PSL tunnel 


\section{Model Formulation - General Description}

- Simulates PSL icing tunnel

- Model couples air and cloud particle conservation eqs

- Mass, energy fully coupled

- Air is treated as ideal compressible gas

- Isentropic equations used to solve $\rho_{\text {air }}, \mathrm{v}_{\text {air }}, \mathrm{T}_{\text {air }}, \mathrm{P}$

- Full particle size distributions used

- "air" = humid air = air + vapor

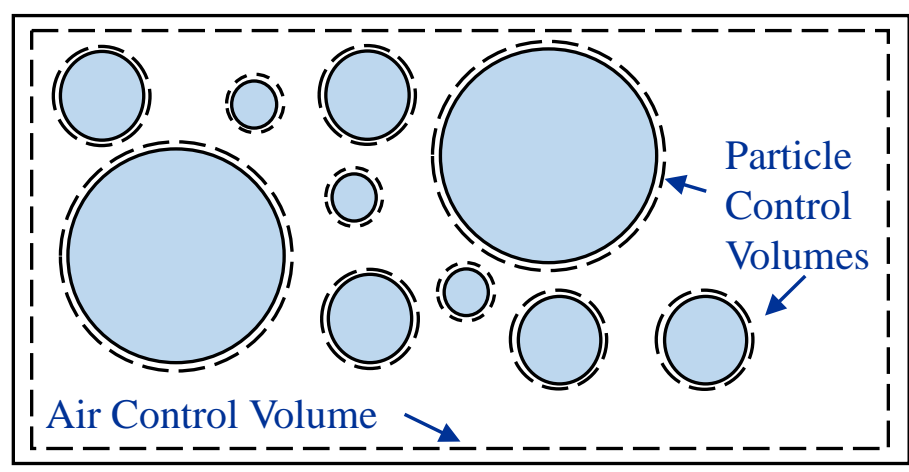




\section{Model Formulation - Assumptions}

- Air and particle flow are steady and one dimensional

- Dry air and water vapor are ideal gases

- Air (air + vapor) is well mixed

- Tunnel is adiabatic and mass is conserved

- Particle size distribution is characterized by a discrete set of diameters

- Particles are evenly spaced

- All particles are perfectly spherical

- Particle aggregation and breakup through collision are negligible

- Particles are injected in the direction of the flow and remain entrained

- Temperature is uniform within the particle

- Mixed phase particles are spatially homogeneous in water/ice content

- Evaporation, condensation occur at the particle surface at particle temperature

- The flow of particles and gas is a continuous stream 


\section{Model Formulation - PSL Description}

\section{Tunnel Controllability}

- $\pm 0.3 \mathrm{kPa}(.05 \mathrm{psia})$

- $\pm 0.5^{\circ} \mathrm{C}\left(1{ }^{\circ} \mathrm{F}\right)$

- $\pm 1 \% \mathrm{RH}$

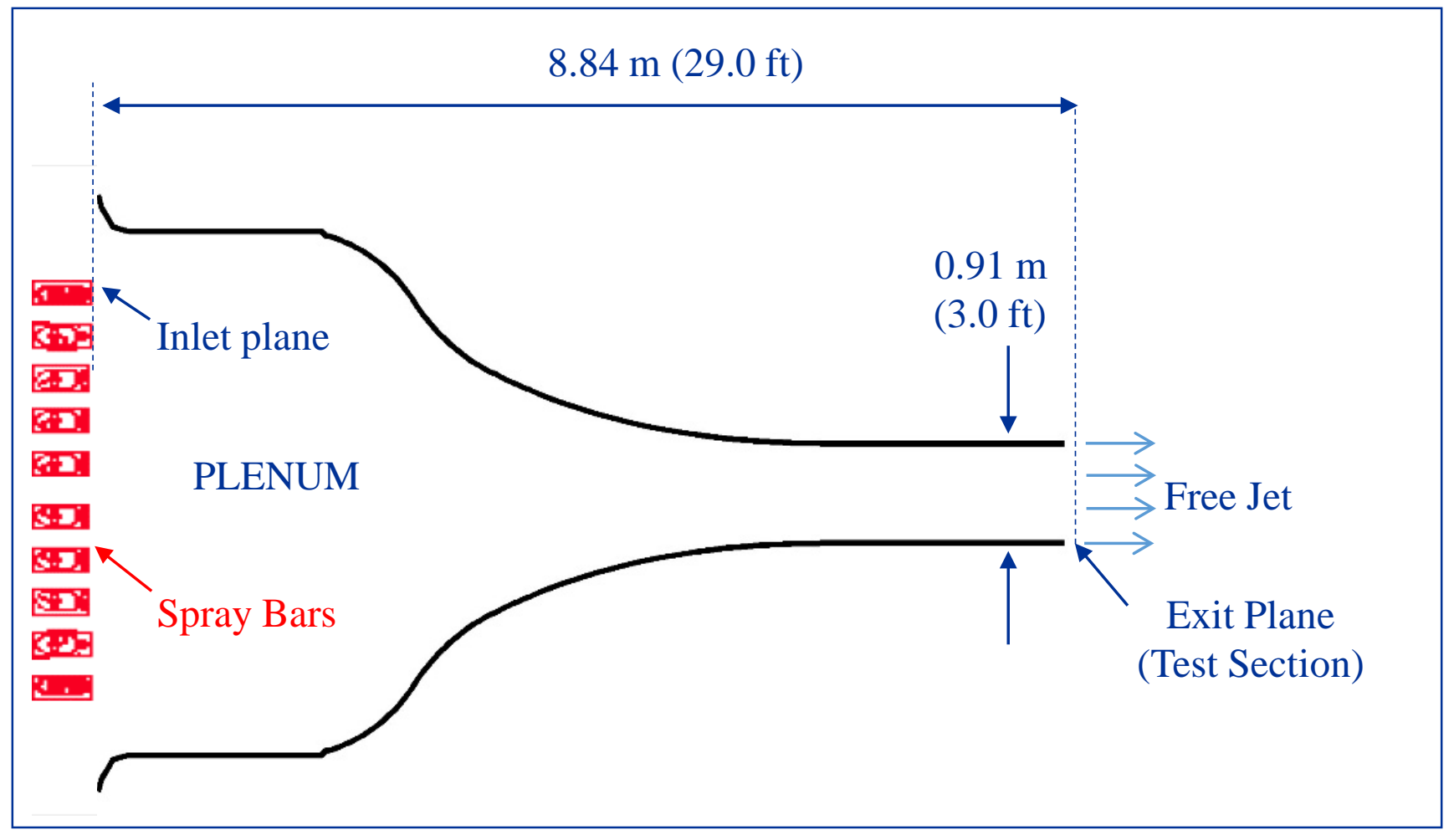


Model Formulation - Experiment Configurations

$$
2 \text { Configurations - May } 2015
$$

Multi-element Probe

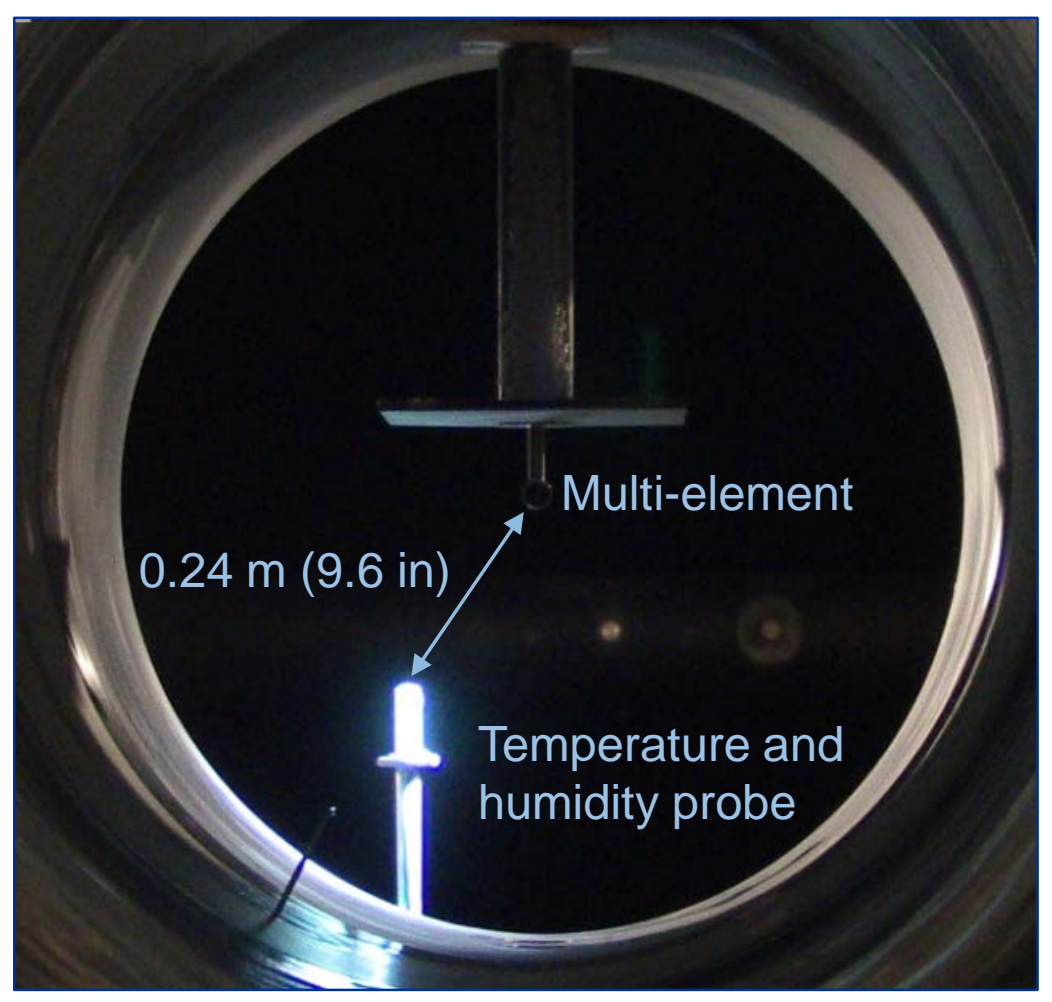

Cloud Droplet Probe

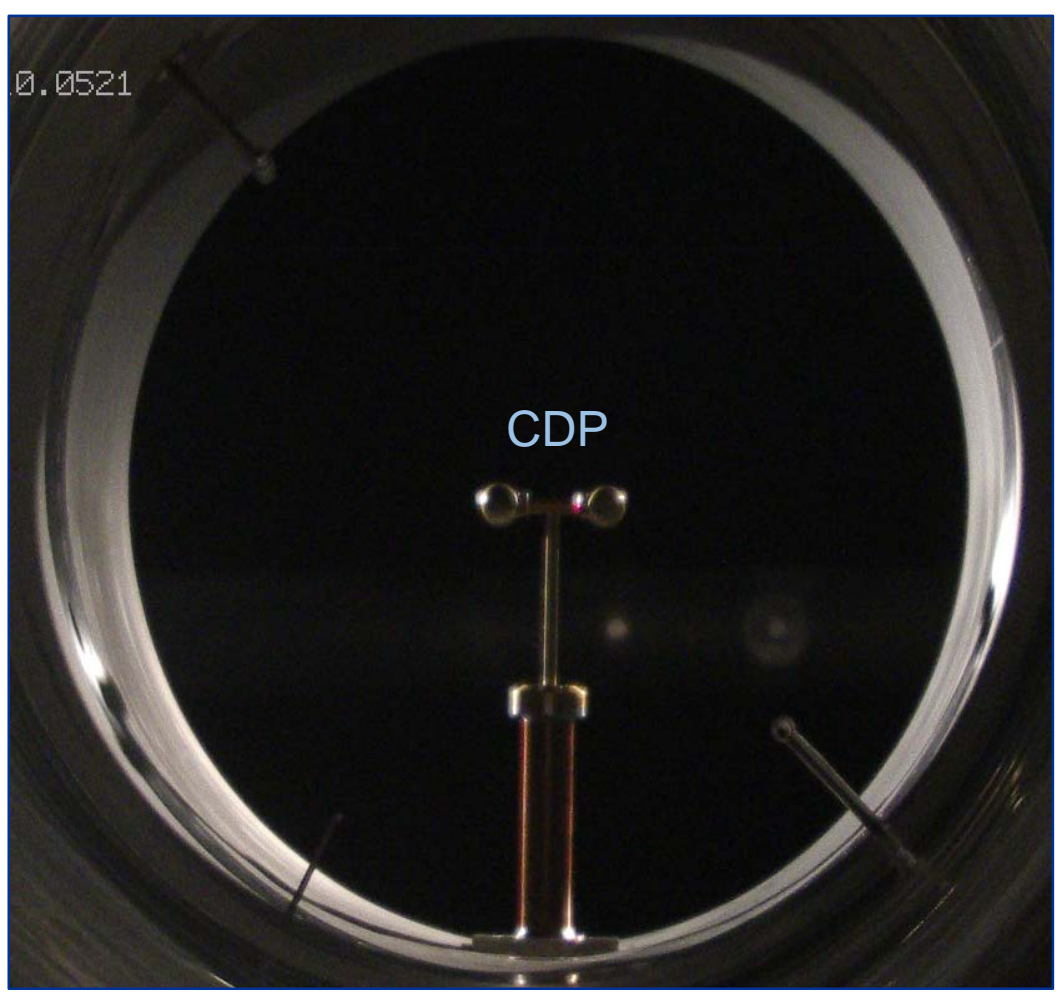




\section{Model Formulation - Differential Expressions}

\section{$\underline{\text { Gas }}$}

$\frac{\partial m_{\text {air }}}{\partial x}$

$\frac{\partial v_{\text {air }}}{\partial x}$

$\frac{\partial T_{a i r}}{\partial x}$

$\frac{\partial \rho_{\text {air }}}{\partial x}$

$\frac{\partial P}{\partial x}$

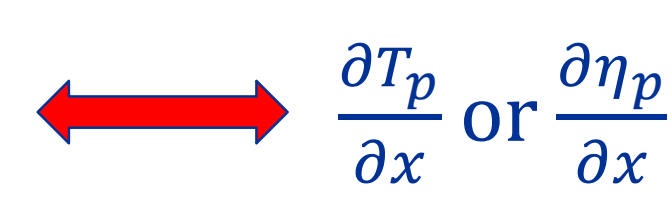

Cloud

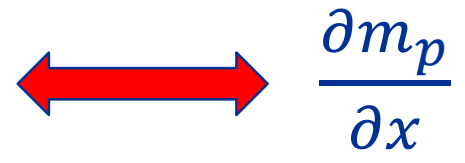

$\longrightarrow \frac{\partial v_{p}}{\partial x}$

$x$

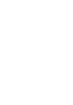

\section{Geometry}

$$
\frac{\partial A}{\partial x}=\text { known }
$$

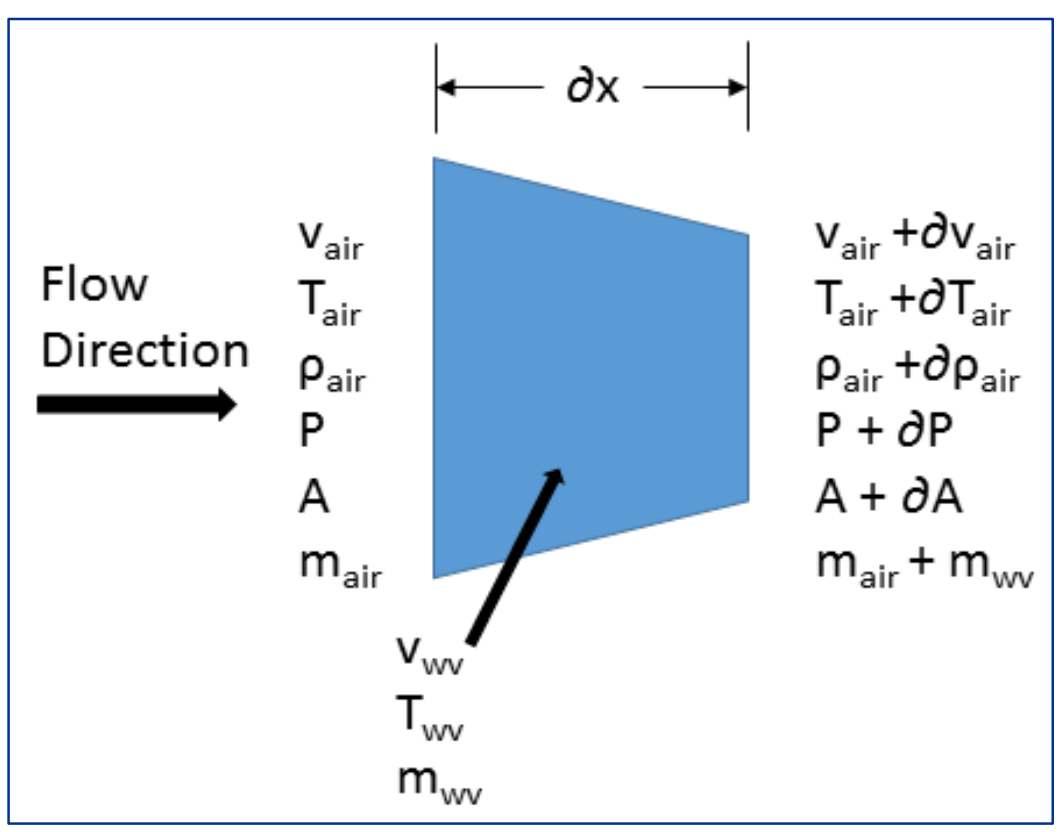




\section{Model Formulation - Differential Expressions}

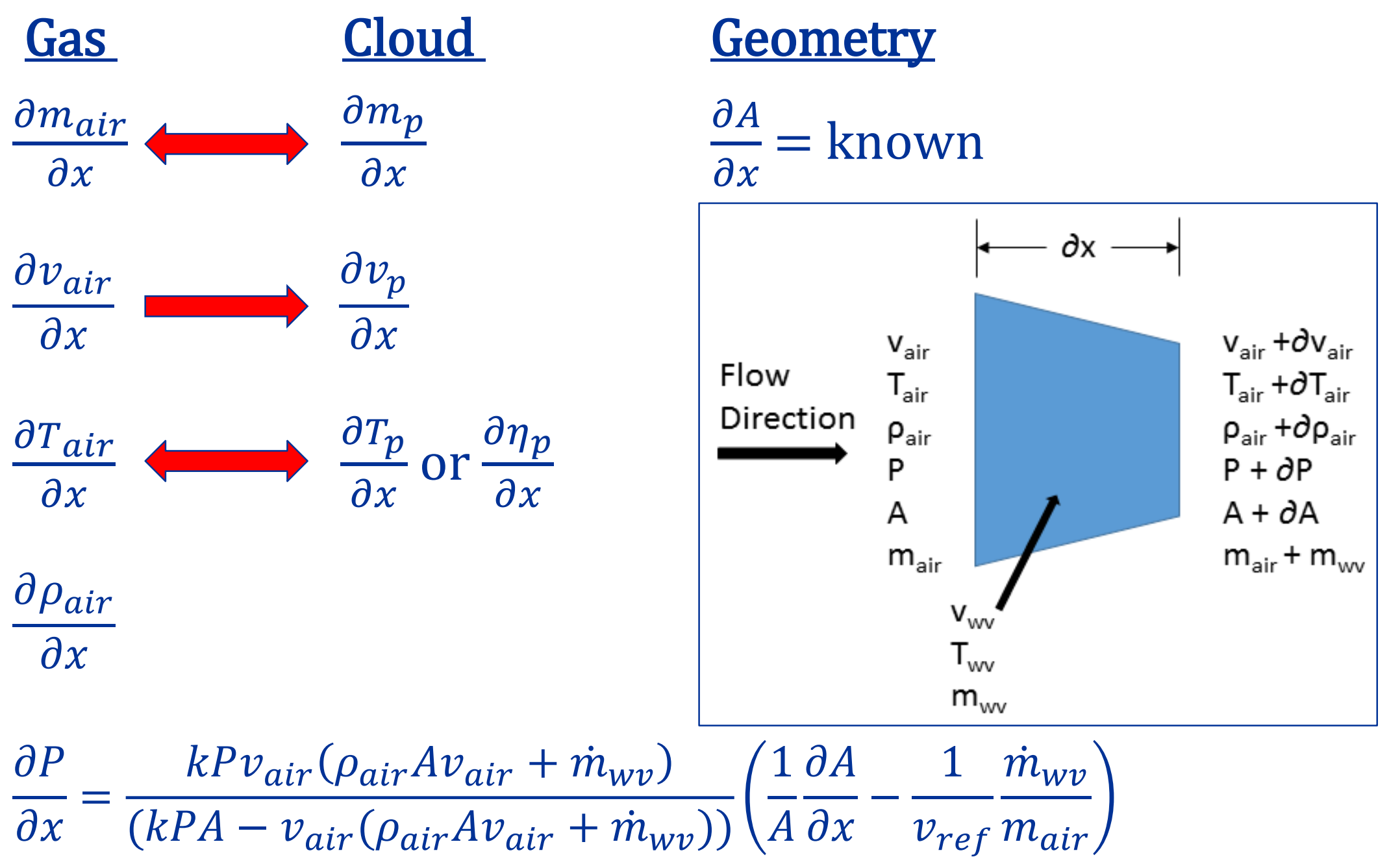




\section{Model Formulation - Algorithm}

- Written in MATLAB version R2015b

- Solves conservation differential equations using built-in ODE45 solver

- Numerical relative and absolute convergence tolerance of $10^{-8}$

- Mass transferred between the gas and particle(s) balanced to 10-15

- Energy transferred between the gas and particle(s) balanced to $10^{-4}$ - Physical accuracy dependent on accuracy of property values $\left(C_{p}, L_{\text {heat }}\right.$, etc.) 


\section{Sample Simulation}

\begin{tabular}{|c|c|}
\hline \multicolumn{2}{|c|}{ Test Conditions } \\
\hline$T_{0}(\mathrm{~K})$ & 277.3 \\
\hline$R H_{0}(\%)$ & 10.8 \\
\hline$P_{0}(\mathrm{kPa})$ & 87.5 \\
\hline$V_{e}(\mathrm{~m} / \mathrm{s})$ & 85 \\
\hline$T W C_{\text {bulk }}\left(\mathrm{g} / \mathrm{m}^{3}\right)$ & 2.3 \\
\hline$T_{\text {water }}(\mathrm{K})$ & 280.4 \\
\hline$M V D(\mu \mathrm{m})$ & 42 \\
\hline$T$ Wb $_{0}(\mathrm{~K})$ & 269.3 \\
\hline
\end{tabular}

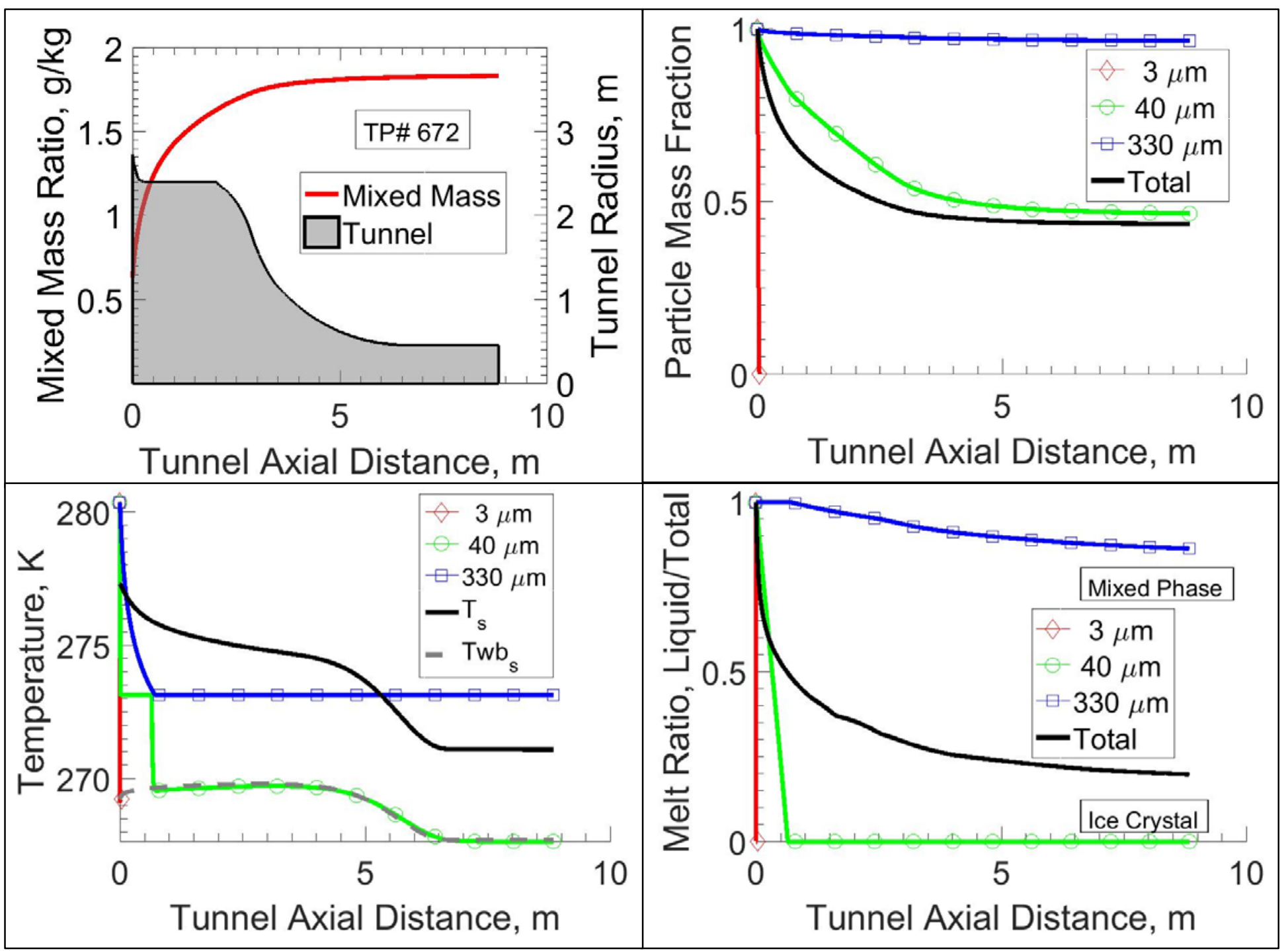

\begin{tabular}{|c|c|}
\hline \multicolumn{2}{|c|}{ Model Results } \\
\hline Res. Time $(\mathrm{s})$ & 1.26 \\
\hline$\Delta T_{0, \mathrm{e}}(\mathrm{K})$ & -2.7 \\
\hline$\Delta \omega_{\mathrm{e}}(\mathrm{g} / \mathrm{kg})$ & 1.2 \\
\hline$\eta_{\mathrm{e}}$ (melt ratio) & 0.20 \\
\hline $\mathrm{TWC}_{\mathrm{e}}\left(\mathrm{g} / \mathrm{m}^{3}\right)$ & 1.0 \\
\hline$\Delta \mathrm{Twb}_{0, \mathrm{e}}(\mathrm{K})$ & 0.5 \\
\hline
\end{tabular}




\section{Model/Experiment Comparison - $T W C_{\text {bulk }}$ Sweep}

\begin{tabular}{|c|c|}
\hline \multicolumn{2}{|c|}{ Target Conditions } \\
\hline$v_{e}(\mathrm{~m} / \mathrm{s})$ & 85 \\
\hline$P_{0}(\mathrm{kPa})$ & 87.3 \\
\hline$T_{0}\left({ }^{\circ} \mathrm{C}\right)$ & 4.2 \\
\hline$R H_{0}(\%)$ & 10 \\
\hline$M V D(\mu \mathrm{m})$ & 40 \\
\hline$T_{\text {water }}\left({ }^{\circ} \mathrm{C}\right)$ & 7 \\
\hline
\end{tabular}
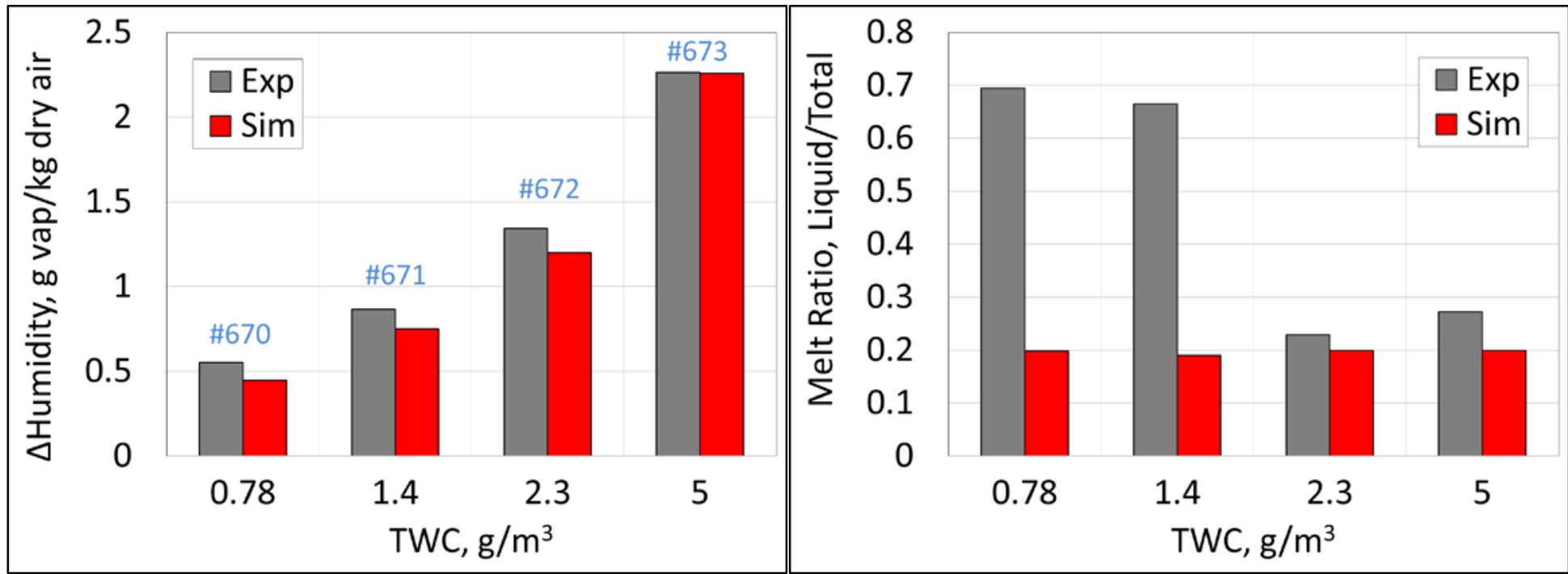

\section{Takeaways:}

- Bulk vs Point

- $\Delta \omega_{\% \text { diff }} \sim 30 \%$

- $\Delta T_{\% \text { diff }} \sim 30 \%$

- $\Delta T w b_{0, e}=$ slight increase

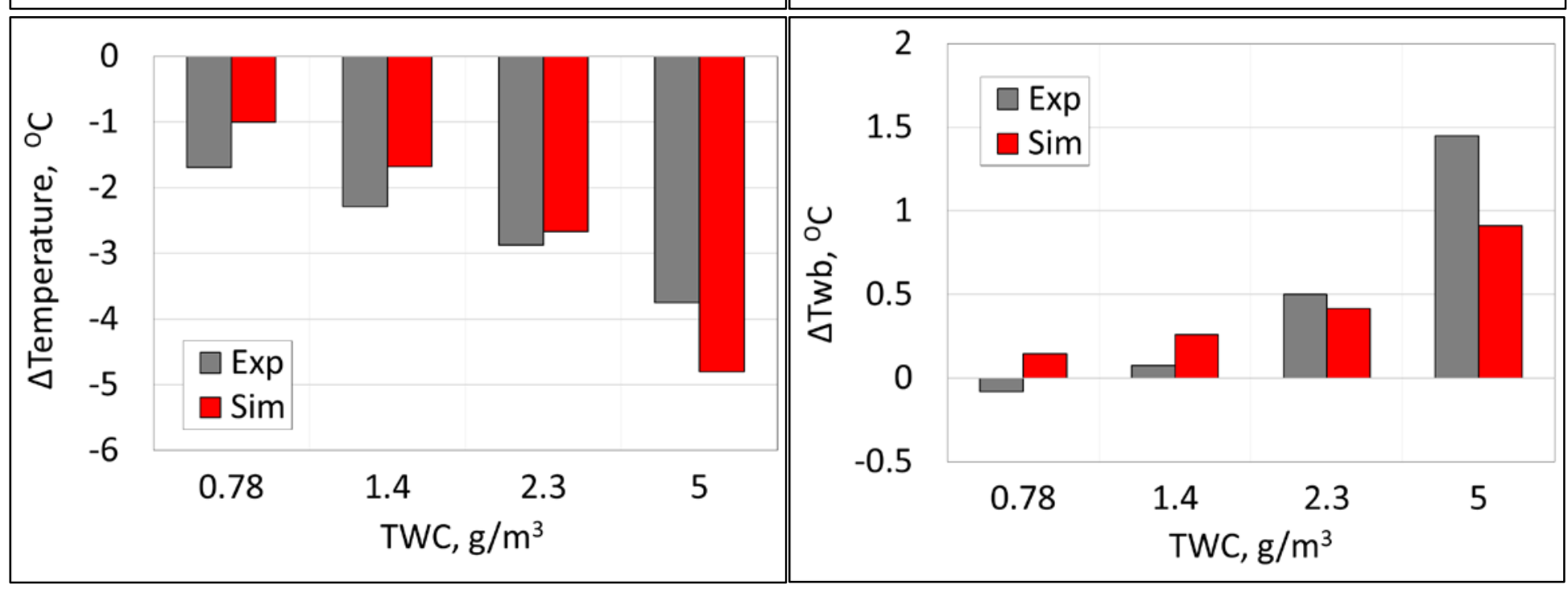




\section{Model/Experiment Comparison - RH Sweep}

\begin{tabular}{|c|c|}
\hline \multicolumn{2}{|c|}{ Target Conditions } \\
\hline$V_{e}(\mathrm{~m} / \mathrm{s})$ & 85 \\
\hline$P_{0}(\mathrm{kPa})$ & 87.2 \\
\hline$T_{0}\left({ }^{\circ} \mathrm{C}\right)$ & 6.6 \\
\hline$T W C_{\text {bulk }}\left(\mathrm{g} / \mathrm{m}^{3}\right)$ & 1.0 \\
\hline$M V D(\mu \mathrm{m})$ & 15 \\
\hline$T_{\text {water }}\left({ }^{\circ} \mathrm{C}\right)$ & 7 \\
\hline
\end{tabular}

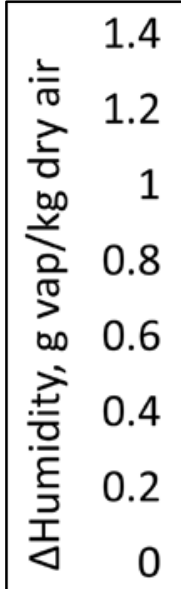

\#667

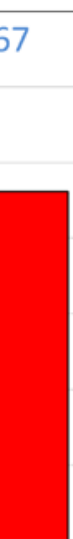

10

Relative Humidity, \%

\begin{tabular}{|c|c|c|}
\hline \multicolumn{3}{|c|}{ Wet Bulb Temps } \\
\hline$R H_{0}$ & $T_{w} b_{0}$ & $T w b_{s}$ \\
\hline$(\%)$ & $\left({ }^{\circ} \mathrm{C}\right)$ & $\left({ }^{\circ} \mathrm{C}\right)$ \\
\hline 10 & -2.2 & -4.4 \\
\hline 35 & 0.6 & -1.7 \\
\hline 50 & 1.7 & -0.6 \\
\hline
\end{tabular}

\section{Takeaways:}

- $T w b_{0}$ important

- Small RH window

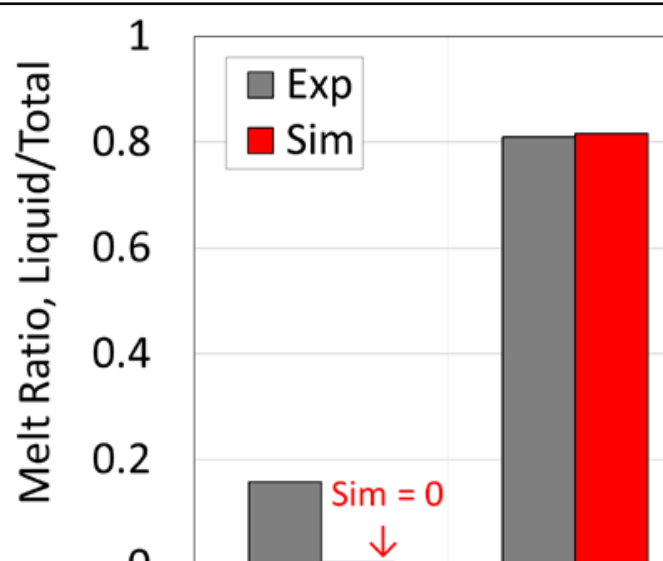

10

35

Relative Humidity, \%
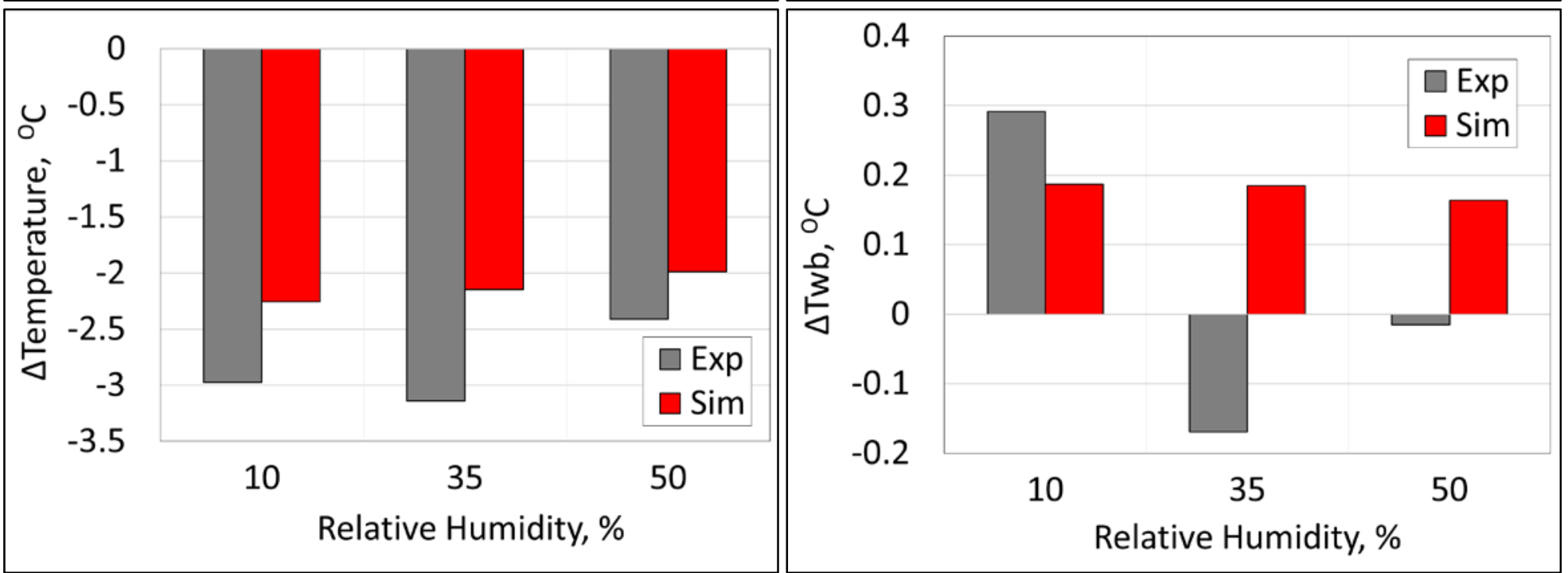


\section{Model/Experiment Comparison $-T_{\text {water }}$ Sweep}

\begin{tabular}{|c|c|}
\hline \multicolumn{2}{|c|}{ Target Conditions } \\
\hline$V_{e}(\mathrm{~m} / \mathrm{s})$ & 85 \\
\hline$P_{0}(\mathrm{kPa})$ & 87.2 \\
\hline$T_{0}\left({ }^{\circ} \mathrm{C}\right)$ & 6.6 \\
\hline$R H_{0}(\%)$ & 10 \\
\hline$T W C_{b u l k}\left(\mathrm{~g} / \mathrm{m}^{3}\right)$ & 1.0 \\
\hline$M V D(\mu \mathrm{m})$ & 15 \\
\hline
\end{tabular}
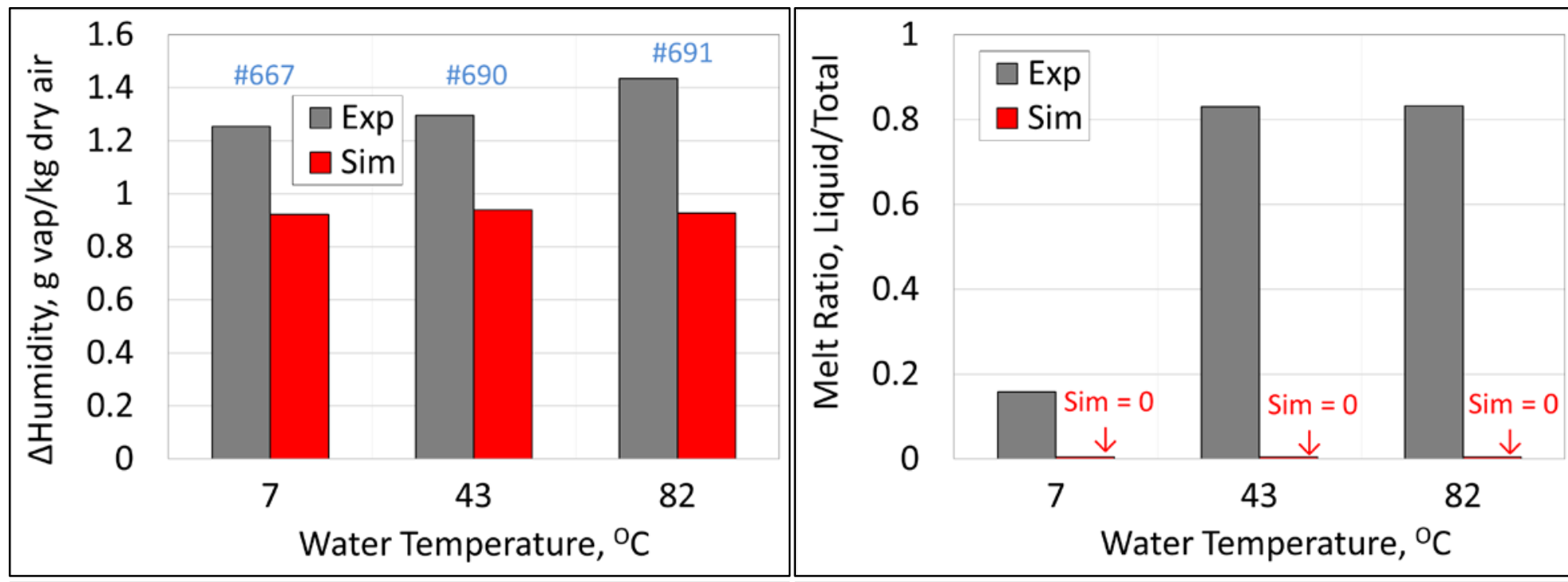

Takeaway:

- Poor melt agreement
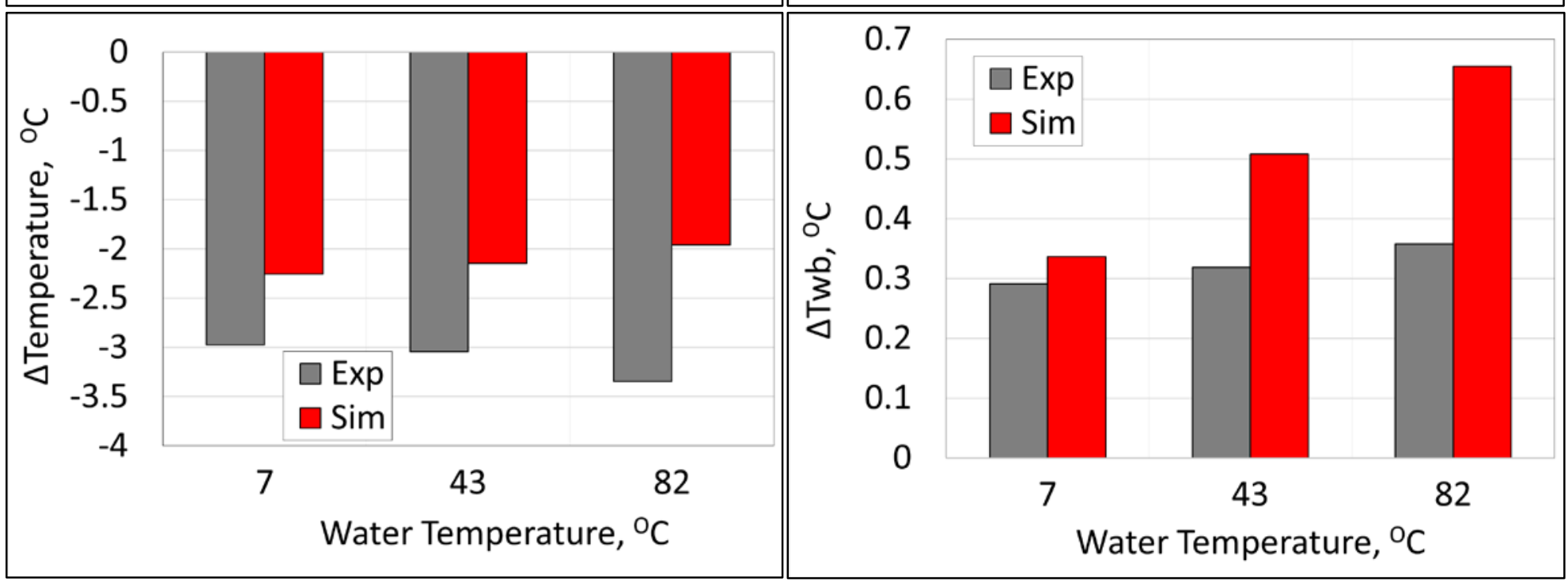


\section{Model/Experiment Comparison - Particle Size}

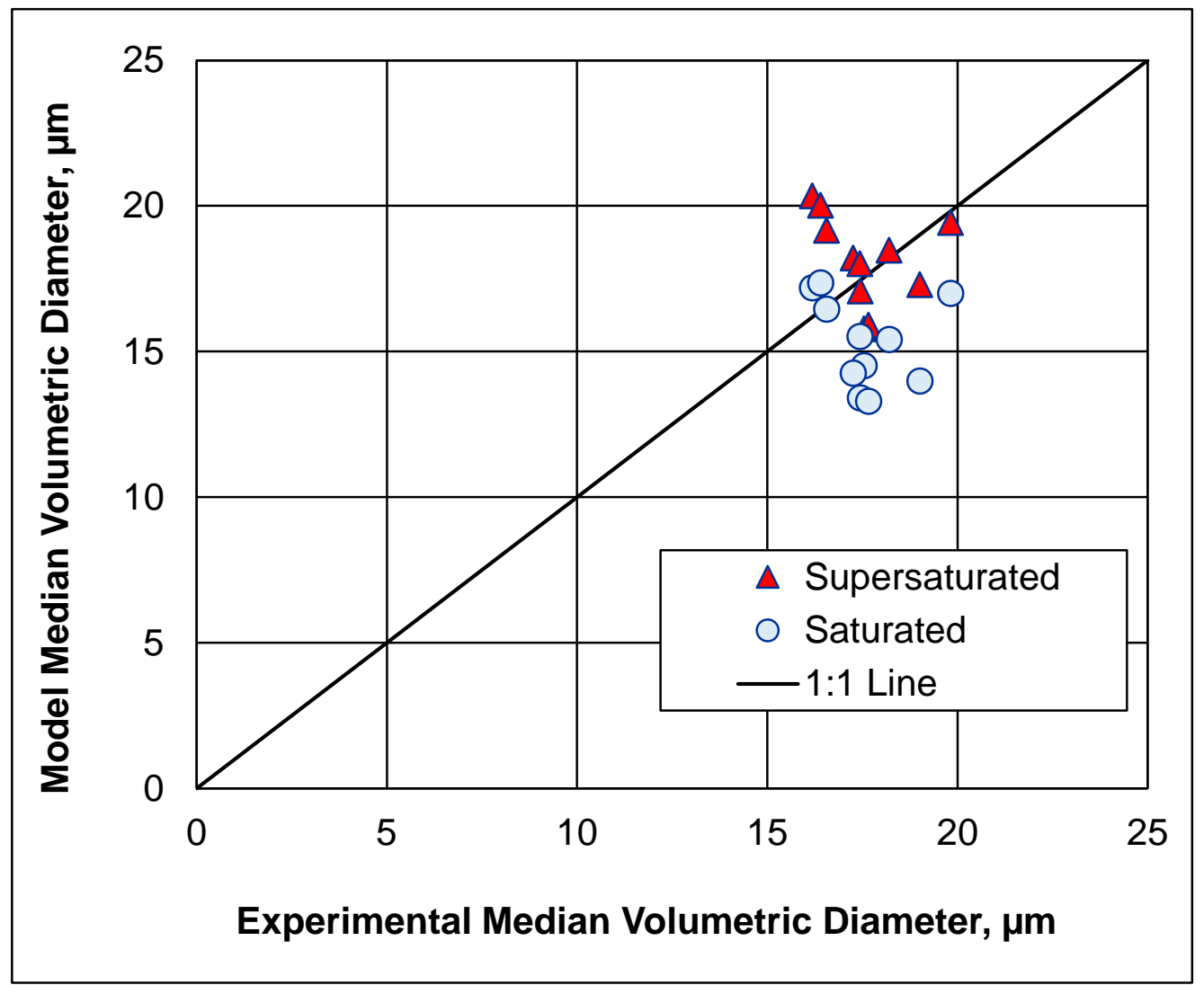

\begin{tabular}{|c|c|c|}
\hline & Min & Max \\
\hline$v_{e}(\mathrm{~m} / \mathrm{s})$ & 68 & 192 \\
\hline$P_{s}(\mathrm{kPa})$ & 32.4 & 84.1 \\
\hline$T_{s}\left({ }^{\circ} \mathrm{C}\right)$ & -29 & 4 \\
\hline$R H_{0}(\%)$ & 40 & 50 \\
\hline$T_{\text {water }}\left({ }^{\circ} \mathrm{C}\right)$ & 7 & 82 \\
\hline$T W C_{\text {bulk }}\left(\mathrm{g} / \mathrm{m}^{3}\right)$ & 0.5 & 1.3 \\
\hline
\end{tabular}

Initial MVD $=15 \mu \mathrm{m}$

Takeaway: Good $M V D_{e}$ agreement for $M V D_{i}=15 \mu \mathrm{m}$ 


\section{Summary}

- Model written to understand Air - Cloud interactions in PSL

- Model predicts to within $30 \%$ of measured changes in humidity and temperature

- Model predicted satisfactorily for melt ratio

- Some disagreement for elevated $T_{\text {water }}$ tests

- Good agreement with particle size measurements

- $\mathrm{Twb}_{0}$ slight increase, important to determine cloud phase

- Model guided development of test matrix for Fundamental Physics of $\mathrm{ICl} 2016$ tests 


\section{Acknowledgements}

- The authors wish to acknowledge the Advance Air Transport Technology Project (AATT) of NASA's Advanced Air Vehicle's program for financial support of this work.

- I would like to thank my Icing Branch colleagues at NASA GRC for technical guidance. 